\title{
Changes in kidney function markers after bariatric surgery in morbidly obese patients
}

\author{
Soon Hyo Kwon ${ }^{(1)}$ \\ Division of Nephrology, Soonchunhyang University Seoul Hospital, Seoul, Republic Korea
}

Obesity is a global health problem that is strongly associated with developing type 2 diabetes mellitus (DM), cardiovascular disease, cerebrovascular accidents, and some malignancies. Additionally, obesity is a major risk factor for the development and progression of chronic kidney disease (CKD) [1].

Bariatric surgery is a proven and effective method for sustaining weight loss, lowering blood pressure, improving glycemic control, and inducing the remission of DM [2]. Additionally, renal parameters may be improved in obese patients after bariatric surgery. A meta-analysis showed that bariatric surgery reduced the unindexed measured glomerular filtration rate (mGFR) in patients with a normal or high GFR, which is suggestive of a return to normofiltration from the hyperfiltration of single nephron GFR [3]. Our previous study also found the GFR in obese patients decreased to an age- and sex-matched normal GFR range after surgery [4]. The presence of albuminuria or proteinuria also decreased after surgeryinduced weight reduction [5].

Is bariatric surgery safe or beneficial in patients with

Received April 21, 2020; Revised May 3, 2020;

Accepted May 4, 2020

Edited by Gheun-Ho Kim, Hanyang University, Seoul, Republic of Korea

Correspondence: Soon Hyo Kwon

Division of Nephrology, Soonchunhyang University Seoul Hospital, 59 Daesagwan-ro, Yongsan-gu, Seoul 04401, Republic Korea.E-mail: ksoonhyo@schmc.ac.kr

Copyright (c) 2020 by The Korean Society of Nephrology

(a) This is an open-access article distributed under the terms of the Creative Commons Attribution Non-Commercial License (http://creativecommons. org/licenses/by-nc-nd/4.0/), which permits unrestricted non-commercial use, distribution, and reproduction in any medium, provided the original work is properly cited.
CKD or end-stage renal disease? Recently, bariatric surgery has been reported to be relatively safe in patients with CKD, although postoperative complications were slightly more frequent in these patients than in the general obese population [6]. In short, bariatric surgery is beneficial for obese patients with CKD who have preserved kidney function. Although several studies have reported that obesity is paradoxically associated with better outcomes in advanced CKD, the presence of morbid obesity in CKD patients is related to the progression of kidney dysfunction [7]. Thus, nephrologists should consider bariatric surgery for morbidly obese patients with CKD.

In this issue of Kidney Research and Clinical Practice (KRCP), Khalil et al [8] showed improvement in kidney function biomarkers after bariatric surgery. Their singlecenter prospective study enrolled 44 obese patients, and the eGFR, albuminuria, and kidney injury molecule (KIM-1) were assessed both before and six months after bariatric surgery. They found that bariatric surgery decreased the serum KIM-1 level and produced albuminuria as well as a lower BMI. The serum KIM-1 level was weakly but significantly correlated with albuminuria and the serum creatinine level before and after surgery. KIM1 is a type 1 transmembrane glycoprotein with two extracellular domains. Following injury, the extracellular domains of KIM-1 separate from the cell surface and enter the systemic circulation and then the urine [9]. Treatment of kidney disease could decrease urinary KIM-1 levels [10]. Thus, the urinary KIM-1 level is a biomarker for early detection of kidney injury and for assessing a patient's therapeutic response to kidney disease. Accumulating evidence indicates that circulating (blood) KIM-1 as well as the urinary KIM-1 levels can be a diagnostic and prognostic biomarker in various kidney diseases, such as 
Table 1. A literature review of the circulating kidney injury molecule-1 (KIM-1) in kidney disease

\begin{tabular}{|c|c|c|c|}
\hline Author (year) & Serum/plasma & Number of patients & Summary of results \\
\hline \multirow{2}{*}{$\begin{array}{l}\text { Sabbisetti et al [9] } \\
\text { (2014) }\end{array}$} & Plasma & 18 & Increased KIM-1 levels in acute kidney injury patients. \\
\hline & & 46 & Increased KIM-1 levels in chronic kidney disease of various etiologies. \\
\hline $\begin{array}{l}\text { Scelo et al [11] } \\
\text { (2018) }\end{array}$ & Plasma & 380 & KIM-1 levels predicted the RCC incidence for up to five years. \\
\hline
\end{tabular}

eGFR, estimated glomerular filtration rate; RCC, renal cell carcinoma.

acute kidney injury, renal cell carcinoma, urinary tract infection, and diabetic nephropathy (Table 1) $[9,11,12]$. We found that the urinary KIM-1 was elevated in obesity but decreased following bariatric surgery [10]. Glomerular hyperfiltration, high blood pressure, and concomitant DM in obese patients may trigger tubular cell injury. Consistent with this information, the authors found that circulating KIM-1 levels decreased after weight reduction in association with improved glycosylated hemoglobin levels.

Interestingly, the reduction in the circulating KIM-1 level was greater in obese patients without albuminuria than it was in those with microalbuminuria [8]. The authors assumed that albuminuria would represent a more severe degree of kidney injury, and weight reduction after bariatric surgery would not improve kidney function in the albuminuria group. However, the link between microalbuminuria and tubular injury remains unclear. In addition, the levels of circulating KIM- 1 before surgery did not differ according to the presence of albuminuria.

The change in albuminuria in CKD patients after treatment may be a surrogate endpoint for the progression of kidney diseases [13]. Whether the reduction of blood or urine KIM-1 levels due to treatment may lead to favorable renal outcomes is yet to be investigated. Thus, it is unclear whether the considerable reduction of KIM-1 after bariatric surgery would be beneficial in obese patients.

The use of sleeve gastrectomy in bariatric surgery is a crucial strength of this study. This strategy offers important metabolic advantages over other surgical techniques for weight reduction, such as laparoscopic adjustable gastric banding, roux-en-Y gastric bypass, and biliopancreatic diversion with duodenal switch. Different surgeries could variably alter kidney function parameters; this effect is likely due to anatomic and functional differences induced by the specific surgical procedures. In addition, the participants in this study demonstrated preserved eGFRs and no overt albuminuria. A homogenous patient population with mild renal injury might have produced more favorable results in this study.

However, this investigation did not measure the urinary KIM-1 level in obese patients. The assessment of urinary and circulating KIM-1 levels both before and after surgery may have revealed more precise KIM-1 behavior according to the patients' BMI status. Additional large prospective studies are warranted to determine if different surgical procedures may influence the levels of urinary and circulating KIM-1 and whether the degree of KIM-1 reduction would be associated with an improvement in kidney function in obese patients.

In summary, the level of KIM-1 was reduced by sleeve gastrectomy, and bariatric surgery may be associated with improvement of renal tubular injury in obese patients. Taken together, the role of tubular injury in the mechanisms of obesity-related renal outcomes needs to be determined. Further studies are required to determine whether urinary and circulating KIM- 1 levels are earlier biomarkers than microalbuminuria in obesity-induced renal injury.

\section{Conflicts of interest}

The author has no conflicts of interest to declare.

\section{References}

[1] GBD 2015 Obesity Collaborators, Afshin A, Forouzanfar $\mathrm{MH}$, et al. Health effects of overweight and obesity in 195 countries over 25 years. N Engl J Med 2017;377:13-27.

[2] Schauer PR, Bhatt DL, Kirwan JP, et al. Bariatric surgery 
versus intensive medical therapy for diabetes--3-year outcomes. N Engl J Med 2014;370:2002-2013.

[3] Navaneethan SD, Yehnert H, Moustarah F, Schreiber MJ, Schauer PR, Beddhu S. Weight loss interventions in chronic kidney disease: a systematic review and meta-analysis. Clin J Am Soc Nephrol 2009;4:1565-1574.

[4] Lee S, Park S, Kwak MK, Kang HR, Kim Y, Kwon SH. Predictors of postoperative eGFR change and resolution of hyperfiltration in obese patients following bariatric surgery. Surg Obes Relat Dis 2017;13:1353-1360.

[5] Afshinnia F, Wilt TJ, Duval S, Esmaeili A, Ibrahim HN. Weight loss and proteinuria: systematic review of clinical trials and comparative cohorts. Nephrol Dial Transplant 2010;25:1173-1183.

[6] Cohen JB, Tewksbury CM, Torres Landa S, Williams NN, Dumon KR. National postoperative bariatric surgery outcomes in patients with chronic kidney disease and endstage kidney disease. Obes Surg 2019;29:975-982.

[7] Rhee CM, Ahmadi SF, Kalantar-Zadeh K. The dual roles of obesity in chronic kidney disease: a review of the current literature. Curr Opin Nephrol Hypertens 2016;25:208-216.

[8] Khalil R, Elghadban H, Abdelsalam M, Tawfik M. Kidney injury molecule-1: a potential marker of renal recovery af- ter laparoscopic sleeve gastrectomy. Kidney Res Clin Pract 2020;39:162-171.

[9] Sabbisetti VS, Waikar SS, Antoine DJ, et al. Blood kidney injury molecule- 1 is a biomarker of acute and chronic kidney injury and predicts progression to ESRD in type I diabetes. J Am Soc Nephrol 2014;25:2177-2186.

[10] Lee H, Oh S, Yang W, et al. Bariatric surgery reduces elevated urinary mitochondrial DNA copy number in patients with obesity. J Clin Endocrinol Metab 2019;104:2257-2266.

[11] Scelo G, Muller DC, Riboli E, et al. KIM-1 as a blood-based marker for early detection of kidney cancer: a prospective nested case-control study. Clin Cancer Res 2018;24:55945601.

[12] Krzemień G, Turczyn A, Pańczyk-Tomaszewska M, Kotuła I, Demkow U, Szmigielska A. Prognostic value of serum and urine kidney injury molecule-1 in infants with urinary tract infection. Cent Eur J Immunol 2019;44:262-268.

[13] Heerspink HJL, Greene T, Tighiouart H, et al. Change in albuminuria as a surrogate endpoint for progression of kidney disease: a meta-analysis of treatment effects in randomised clinical trials. Lancet Diabetes Endocrinol 2019;7:128-139. 